\title{
Metodologias ativas de ensino-aprendizagem: uso, dificuldades e capacitação entre docentes de curso de Medicina
}

\author{
Active teaching and learning methodologies: use, difficulties and training among medical course teachers
}

Katia Jakovljevic Pudla Wagner ${ }^{1,2}$ (D) $\mid$ katia.wagner@ufsc.br Lourival José Martins Filho² (D) lourivalfaed@gmail.com

\section{RESUMO}

Introdução: No Brasil, as Diretrizes Curriculares Nacionais para o curso de Medicina apontam que a formação médica deverá usar metodologias que privilegiem a participação ativa do aluno na construção do conhecimento, além de preverem a existência de programas de desenvolvimento e aperfeiçoamento docente no interior dos cursos de Medicina.

Objetivo: Este estudo teve como objetivo avaliar o uso, a dificuldade e a capacitação referentes às metodologias ativas de ensino-aprendizagem (MAP) em docentes do curso de Medicina em dois campi da Universidade Federal de Santa Catarina.

Método: Trata-se de estudo transversal com coleta de dados por meio de questionário em formato on-line, em que se pesquisaram variáveis demográficas, de formação acadêmica, de atuação docente e de MAP, incluindo seu uso na prática pedagógica, dificuldades e realização de capacitação no tema. Foram feitas análises estatísticas com o intuito de verificar diferenças entre as variáveis analisadas.

Resultado: Participaram 63 docentes, com média de idade de 48,2 anos, maioria do gênero feminino (57,1\%), com regime de trabalho de dedicação exclusiva $(61,9 \%)$ e metade da amostra composta por médicos. Apenas um a cada dez professores referiu que não utiliza e/ou nunca utilizou MAP na sua atuação como docente, e metade mencionou ter dificuldade na sua utilização. Quanto à capacitação pedagógica, $54 \%$ referiram ter realizado, dos quais $67,7 \%$ mencionaram que ela não foi suficiente para dar densidade teórica ao processo de ensino-aprendizagem com MAP. Estudo de caso e aprendizagem baseada em problemas foram as MAP mais conhecidas e utilizadas dentro da universidade.

Conclusão: O uso das MAP foi alto, e pouco mais da metade dos docentes tem alguma capacitação no tema. Considerando que a maioria dos docentes apontou dificuldades no seu uso com os discentes, faz-se necessário planejar a metodologia, a carga horária ou os temas das capacitações que são oferecidas.

Palavras-chave: Educação Médica; Metodologias Ativas de Ensino-Aprendizagem; Aprendizagem Baseada em Problemas; Educação Superior.

\section{ABSTRACT}

Introduction: In Brazil, the National Curriculum Guidelines for Medicine courses state that medical training should use methodologies that favor the active participation of the student in the construction of knowledge, in addition to providing for the existence of teacher development and improvement programs within Medical courses.

Objective: To evaluate the use, difficulty and training related to Active Teaching and Learning Methodologies (MAP) in teachers of the Medicine course in two campuses of the Federal University of Santa Catarina.

Method: Cross-sectional study with data collection through a questionnaire in an online format, researching demographic, academic education, teaching and MAP variables, including its use in pedagogical practice, difficulties and training on the subject. Statistical analyses were performed in order to verify differences among the analyzed variables.

Results: 63 teachers participated, with a mean age of 48.2 years, mostly female (57.1\%), who worked exclusively for the university (61.9\%) and half of the sample population was composed of physicians. Only one in ten teachers reported that they do not use and/or never used MAP in their work as a teacher, and half reported having difficulty in using it. As for the pedagogical training, 54\% reported having performed it, and among these, $67.7 \%$ reported that it was not enough to give theoretical density to the teaching-learning process with MAP. Case study and problem-based learning were the best-known MAPs and used within the university.

Conclusion: The use of MAP was high and just over half of the teachers reported some training in the subject. Considering that most teachers pointed out difficulties in its use with students, it is necessary to plan the methodology, workload or themes of the training that are offered.

Keywords: Medical education; Active Methodologies; Problem-Based Learning; Higher education.

${ }^{1}$ Universidade Federal de Santa Catarina, Curitibanos, Santa Catarina, Brasil.

2 Programa de Pós-graduação em Educação (PPGE). Universidade do Estado de Santa Catarina, Florianópolis, Santa Catarina, Brasil.

Editora-chefe: Rosiane Viana Zuza Diniz.

Editor associado: Roberto Zonato Esteves.

Recebido em 22/10/21; Aceito em 02/12/21.

Avaliado pelo processo de double blind review. 


\section{INTRODUÇÃO}

A educação médica no Brasil sofreu alterações ao longo do tempo no que se refere aos conteúdos e às metodologias de ensino. No ano de 2001, criaram-se as primeiras Diretrizes Curriculares Nacionais (DCN) para o curso de Medicina que serviram como guia para todas as escolas médicas. No entanto, algumas mudanças se fizeram necessárias por conta da transformação do perfil do médico que se espera formar para lidar com a saúde da população brasileira, e, nesse sentido, em 2014 foram divulgadas as novas DCN ${ }^{1,2}$.

As DCN de 2014 surgem com o propósito de promover uma formação médica mais geral, humanista e crítica, com capacidade para atuar nos diferentes níveis de atenção à saúde. Entre as mudanças, as DCN definem que a formação médica deverá usar metodologias que privilegiem a participação ativa do aluno na construção do conhecimento, além de preverem a existência de programas de desenvolvimento e aperfeiçoamento docente no interior dos cursos de Medicina².

As metodologias ativas de ensino-aprendizagem (MAP) citadas nas novas DCN consistem em uma forma de ensino em que os alunos atuam como protagonistas, sendo estimulados a participar do processo de aprendizagem de forma mais direta. Elas diferem do modelo tradicional de educação que coloca o professor como foco principal do ensino, em que ele transmite conhecimento aos discentes ${ }^{3,4}$.

O papel do docente, quando os currículos são baseados em metodologias ativas, exige outra relação com o processo de aprendizagem das pessoas ${ }^{5}$. No entanto, a formação das carreiras da área da saúde historicamente foi baseada nas metodologias de ensino tradicionais, ou seja, a maioria dos docentes teve como experiência de ensino uma metodologia distinta da preconizada pelas atuais $\mathrm{DCN}^{4}$.

Nesse sentido, implementar um currículo baseado em MAP envolve desafios que abrangem questões tanto estruturais das universidades quanto concepções pedagógicas dos alunos e professores ${ }^{6-8}$. No que se refere aos docentes, capacitação nessa temática se faz importante quando se visa formar um profissional ativo e preparado?.

Os estudos que focam o desenvolvimento docente voltado às MAP foram realizados principalmente em países de renda alta, que possuem realidade diferente do cenário brasileiro de educação. Ao analisar o binômio capacitação de docentes versus MAP para atuar em cursos de Medicina, uma revisão sistemática apontou que a literatura sobre o tema ainda é escassa no país ${ }^{10}$. Outro estudo que fez uma síntese da literatura publicada apenas no Brasil sobre docência na área da saúde constatou que a maioria dos estudos foi realizada na Região Sudeste do país e que o debate sobre MAP ainda é necessário ${ }^{11}$. Assim, o objetivo do presente estudo foi avaliar o uso, a dificuldade e a capacitação referentes às MAP em docentes do curso de Medicina, em dois campi da Universidade Federal de Santa Catarina (UFSC).

\section{MÉTODO}

Realizou-se um estudo transversal com dados quantitativos. Participaram do estudo docentes do curso de Medicina da UFSC de dois diferentes campi: Florianópolis e Araranguá.

A UFSC, sediada no município de Florianópolis, em Santa Catarina, é uma autarquia federal de ensino superior fundada em 1960 e que possui estrutura física de cinco campi (Florianópolis, Curitibanos, Joinville, Araranguá e Blumenau) ${ }^{12}$. Dois deles (Florianópolis e Araranguá) tinham curso de Medicina em andamento nos semestres relativos ao ano de 2020.

Em Florianópolis, o curso de Medicina tem turmas desde o ano de 1960, e seu currículo passou por mudanças ao longo do tempo. Em Araranguá, o curso de Medicina teve início no segundo semestre de 2018, planejado já no formato preconizado pelas DCN de 2014.

Participaram do estudo docentes que atuaram no curso de Medicina da UFSC nos dois campi e que ministraram disciplinas em pelo menos um dos dois semestres relativos ao ano de 2020, que, por conta da pandemia da coronavirus disease 2019 (Covid-19) e da mudança no calendário acadêmico, acabaram sendo durante os anos de 2020 e 2021. A lista dos docentes elegíveis para o estudo foi solicitada a cada um dos 13 departamentos que atuavam na Medicina nos dois campi. Excluíram-se os docentes que estavam em licença no período que compreendeu a coleta de dados. Um fluxograma com os docentes elegíveis e a taxa de resposta em cada um dos campi pode ser visto na Figura 1.

O convite para participar da pesquisa foi feito por e-mail de forma individual, e realizaram-se, em média, três contatos com cada um dos participantes durante os meses de junho e julho de 2021. Enviou-se um questionário em formato on-line via formulário Google Forms que continha perguntas abertas (idade, ano de ingresso na instituição e anos de atuação como docente) e fechadas. Inicialmente foram coletados dados de variáveis demográficas (idade, gênero, cor da pele), de formação acadêmica (curso de formação na graduação, maior grau de titulação) e de atuação docente (regime de trabalho, campus de atuação na UFSC, ano de ingresso na instituição, anos de atuação como docente e área de atuação dentro do curso de Medicina). O segundo bloco do questionário continha perguntas relacionadas às MAP na prática pedagógica. $O$ questionário continha as seguintes perguntas: "Você utiliza ou já utilizou metodologias ativas de ensino-aprendizagem na sua atuação como docente?"; "Você tem alguma dificuldade 
em utilizar metodologias ativas na sua atuação como docente?"; "Você possui alguma capacitação pedagógica em metodologias ativas de ensino-aprendizagem?"; "Você considera que a capacitação recebida foi suficiente para dar densidade teórica ao processo de ensino-aprendizagem com metodologias ativas?". A opções de todas as respostas eram "sim" ou "não". Aos que responderam "sim" à questão da realização de capacitação, indagou-se quem havia oferecido a capacitação, cujas opções de resposta eram: UFSC; outra instituição de ensino em que o participante tem/tinha vínculo empregatício; ele mesmo se inscreveu em um curso por conta própria ou outra. Também foi perguntado "Como avalia a sua própria capacitação pedagógica em metodologias ativas de ensino-aprendizagem?", cujas opções de resposta eram: ótima, boa, regular, ruim e péssima. Para análise dos tipos de MAP conhecidas e utilizadas entre os docentes, utilizaram-se os dados das questões "Você conhece ou já ouviu falar de alguma das seguintes metodologias ativas de ensino-aprendizagem?" e "Qual(is) metodologia(s) ativa(s) de ensino- aprendizagem você utiliza e/ou já utilizou?", as quais tinham como opções de resposta: estudo de caso, problematização, peer education (educação entre pares), aprendizagem baseada em equipes (team-based learning - TBL), aprendizagem baseada em projetos (project-based learning - $\mathrm{PjBL}$ ), aprendizagem baseada em problemas (problem-based learning - $\mathrm{PBL}$ ), laboratórios de simulação, aula invertida, gamificação e outras; e mais de uma opção poderia ser assinalada. Para melhor visualização do questionário referente às MAP, o Quadro 1 apresenta as questões e opções de resposta.

Os dados foram exportados do Excel para o programa estatístico Stata versão 15.1. Primeiramente, categorizaram-se os dados e realizou-se análise descritiva dos dados com valores absolutos e relativos para cada uma das variáveis estudadas. Foram testadas associações das variáveis demográficas, de formação acadêmica e atuação docente com as variáveis relativas às MAP, por meio de teste de qui-quadrado de Pearson ou exato de Fisher. Apenas a variável idade apresenta-se de forma contínua, e, para verificar diferenças entre as médias, utilizou-se o teste $\mathrm{t}$ de Student. Para ambos os testes, foram considerados estatisticamente significativos valores de $p \leq 0,05$.

O projeto foi submetido ao Comitê de Ética em Pesquisa com Seres Humanos da UFSC e aprovado sob Parecer $\mathrm{n}^{\circ}$ 4.706.595, de 12 de maio de 2021. Todos os participantes deram aceite no Termo de Consentimento Livre e Esclarecido antes do início do preenchimento do questionário on-line.

\section{RESULTADOS}

Participaram do estudo 63 docentes da UFSC: 52 do campus de Florianópolis e 11 do campus de Araranguá. A taxa de resposta do questionário foi de 32,6\%, e maiores detalhes sobre a seleção dos participantes podem ser vistos na Figura 1.

A Tabela 1 apresenta a caracterização da amostra em relação às variáveis demográficas, de formação acadêmica e de atuação docente. A média de idade dos docentes foi de 48,2 anos ( $D P=10,8)$, e a maioria era do gênero feminino $(57,1 \%)$ e de cor da pele branca (88,7\%). Metade da amostra foi composta por médicos $(50,0 \%)$, e a maioria absoluta da amostra tinha

Quadro 1. Questionário aplicado aos docentes com as perguntas e opções de resposta referentes às metodologias ativas de ensino-aprendizagem Florianópolis e Araranguá, SC, 2021.

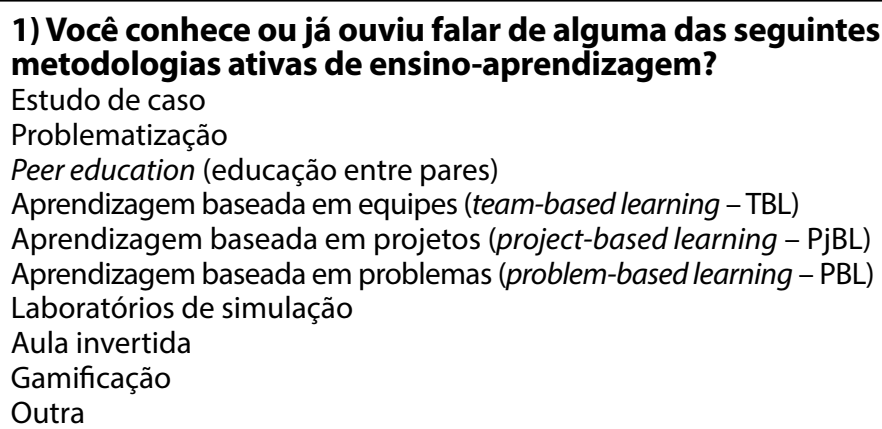

2) Você utiliza ou já utilizou metodologias ativas de ensino-aprendizagem na sua atuação como docente? Sim

Não

3) Qual(is) metodologia(s) ativa(s) de ensinoaprendizagem você utiliza e/ou já utilizou? Opções de resposta iguais à pergunta 1 .

4) Você tem alguma dificuldade em utilizar metodologias ativas na sua atuação como docente?

Sim

Não

5) Você possui alguma capacitação pedagógica em metodologias ativas de ensino-aprendizagem?

Sim

Não

6) A capacitação da qual você participou em metodologias ativas de ensino-aprendizagem foi:

Oferecida pela UFSC

Oferecida por outra instituição de ensino na qual eu tenho/tinha vínculo empregatício

Eu mesmo(a) me inscrevi em um curso nessa área por conta própria Outro

7) Você considera que a capacitação recebida foi suficiente para dar densidade teórica ao processo de ensino-aprendizagem com metodologias ativas? Sim

Não

8) Como avalia a sua própria capacitação pedagógica em metodologias ativas de ensino-aprendizagem?

Ótima

Boa

Regular

Ruim

Péssima

Fonte: Elaborado pelos autores. 
doutorado (87,3\%). Dois a cada três docentes tinham 11 anos ou mais de atuação como docente, e a maioria (61,9\%) tinha como regime de trabalho dedicação exclusiva. Em relação à área de atuação dentro do curso de Medicina, um terço da amostra atuava nas áreas básicas, seguidas da clínica médica e saúde coletiva. Quando se comparou a amostra entre os dois campi, observaram-se diferenças em relação à idade, com média menor no campus de Araranguá, ao ano de término da graduação e aos anos de atuação como docente: os professores de Araranguá se graduaram mais recentemente e têm, em sua maioria, menos anos de experiência docente. Em relação ao ano de ingresso na UFSC, também se verificaram diferenças, visto que em Araranguá o curso abriu em 2018, e todos foram contratados no período mais recente analisado.

Figura 1. Fluxograma da seleção dos docentes participantes.

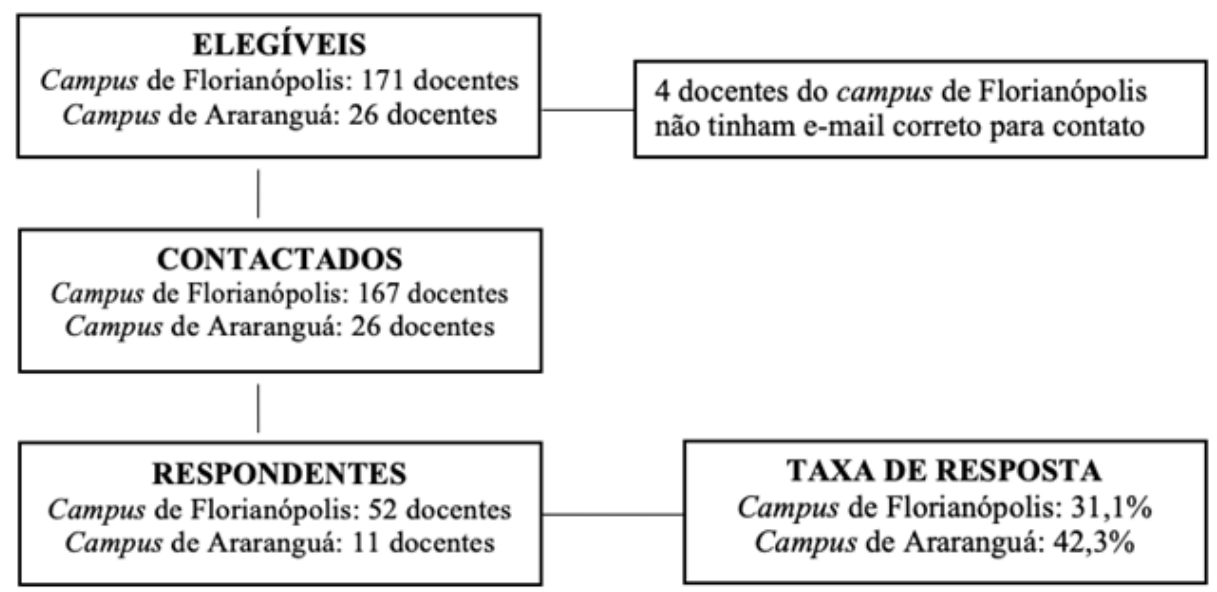

Fonte: Elaborada pelos autores.

Tabela 1. Caracterização da amostra dos docentes do curso de Medicina dos campi de Florianópolis e Araranguá da Universidade Federal de Santa Catarina - Florianópolis e Araranguá, SC, 2021.

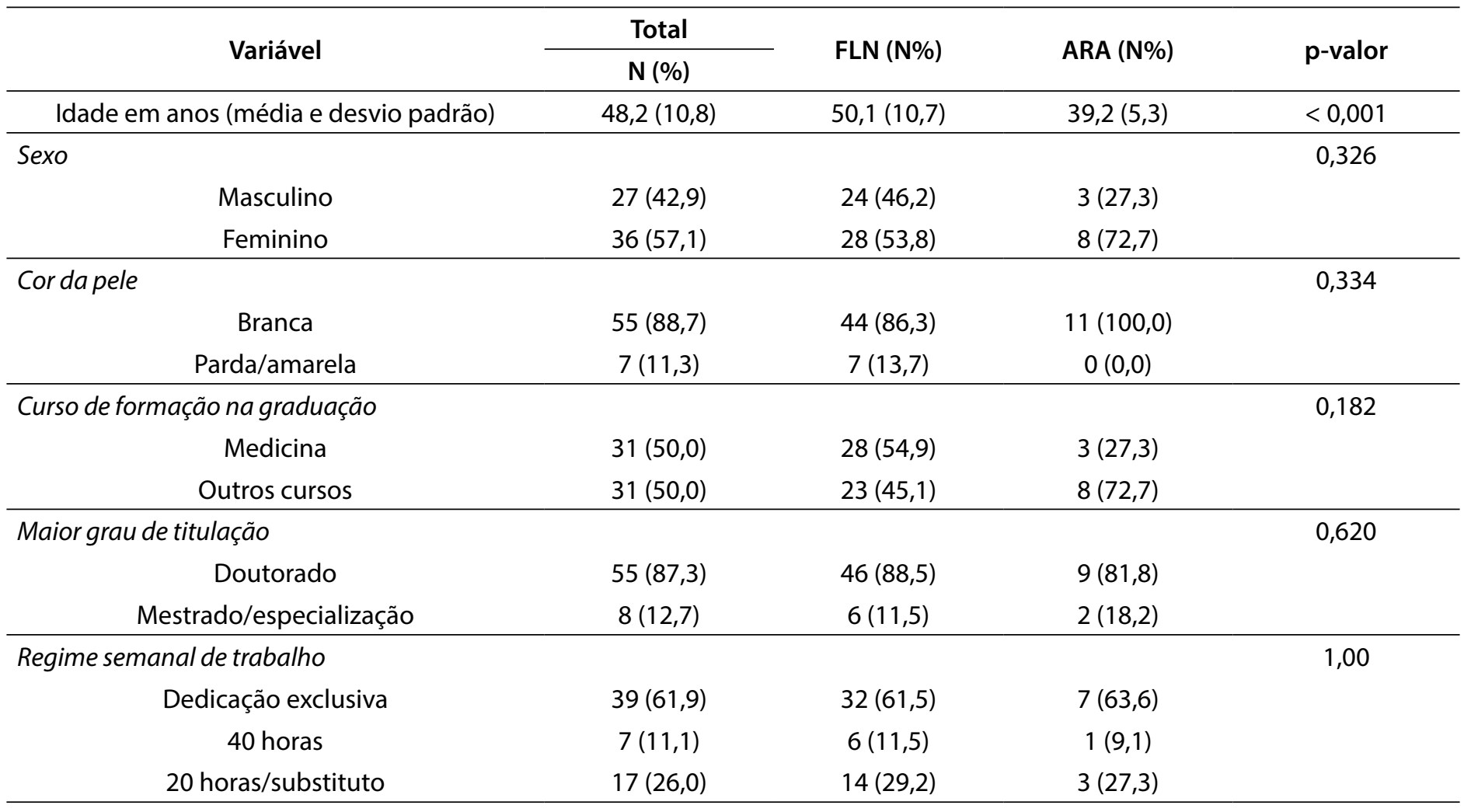


Tabela 1. (Continuação) Caracterização da amostra dos docentes do curso de Medicina dos campi de Florianópolis e Araranguá da Universidade Federal de Santa Catarina - Florianópolis e Araranguá, SC, 2021.

\begin{tabular}{ccccc}
\hline Variável & Total & FLN (N\%) & ARA (N\%) & p-valor \\
\cline { 2 - 2 } Área de atuação no curso de Medicina & $\mathbf{N}(\%)$ & & & 0,386 \\
Clínica médica & $10(15,9)$ & $10(19,2)$ & $0(0,0)$ & \\
Cirurgia & $4(6,3)$ & $3(5,8)$ & $1(9,1)$ & \\
Pediatria & $4(6,3)$ & $4(7,7)$ & $0(0,0)$ & \\
Saúde coletiva & $8(12,7)$ & $6(11,5)$ & $2(18,2)$ & \\
Áreas básicas & $23(36,5)$ & $17(32,7)$ & $6(54,5)$ & \\
Ginecologia/obstetrícia & $3(4,8)$ & $2(3,8)$ & $1(9,1)$ & \\
Outras & $11(17,5)$ & $10(19,2)$ & $1(9,1)$ & \\
1976-1989 & & & & 0,007 \\
1990-2009 & $19(30,2)$ & $19(36,5)$ & $0(0,0)$ & \\
2010-2015 & $36(57,1)$ & $29(55,8)$ & $7(63,6)$ & \\
\hline Ano de término da graduacão & $8(12,7)$ & $4(7,7)$ & $4(36,4)$ & \\
De 0 a 5 anos & & & & \\
De 6 a 10 anos & $14(22,2)$ & $8(15,4)$ & $6(54,5)$ & \\
11 ou mais anos & $8(12,7)$ & $6(11,5)$ & $2(18,2)$ & \\
\hline Anos de atuação como docente & $41(65,1)$ & $38(73,1)$ & $3(27,3)$ & \\
\hline 1979-1999 & $15(23,8)$ & $15(28,8)$ & 0,007 \\
2000-2013 & $21(33,3)$ & $21(40,4)$ & $0(0,0)$ & $11(100)$ \\
2014-2021 & $27(42,9)$ & $16(30,8)$ & & \\
\hline Ano de ingresso na UFSC & & & & \\
\hline
\end{tabular}

Fonte: Elaborada pelos autores.

Apenas um a cada dez docentes referiu que não utiliza e/ou nunca utilizou MAP na sua atuação como docente. No entanto, destaca-se que metade da amostra $(50,9 \%)$ referiu ter dificuldade na sua utilização. Quando questionados se haviam realizado alguma capacitação pedagógica nesse tema, pouco mais da metade da amostra $(54,0 \%)$ referiu que sim, sendo a capacitação oferecida pela própria UFSC como a mais relatada pelos docentes, seguida por aqueles que referiram ter realizado alguma capacitação por conta própria. Entre aqueles que realizaram alguma capacitação pedagógica, $67,7 \%$ referiram que ela não foi suficiente para dar densidade teórica ao processo de ensino-aprendizagem com MAP. Em relação à avaliação da própria capacitação pedagógica em MAP, dois a cada três docentes classificaram como regular, ruim ou péssima (Tabela 2 ).

Em relação aos diferentes tipos de MAP utilizadas na prática docente, destaca-se que a maioria dos docentes $(68,4 \%)$ referiu utilizar três ou mais delas. As MAP cujos docentes referiram mais conhecer foram estudo de caso e PBL (nove em cada dez docentes), sendo essas também as mais utilizadas na prática docente. As outras MAP mais utilizadas pelos docentes foram aula invertida $(47,6 \%)$, problematização $(39,7 \%)$ e laboratórios
Tabela 2. Descrição da amostra segundo variáveis relacionadas às metodologias ativas de ensino-aprendizagem (MAP) na prática pedagógica dos docentes - Florianópolis e Araranguá, SC, 2021.

\begin{tabular}{ccc}
\hline Variável & N & $\%$ \\
\hline Utiliza ou já utilizou MAP na atuação como docente & & \\
Não & 6 & 9,5 \\
Sim & 57 & 90,5 \\
\hline
\end{tabular}

Tem dificuldade em utilizar MAP

\begin{tabular}{ccc} 
Não & 28 & 49,1 \\
Sim & 29 & 50,9 \\
\hline
\end{tabular}

Tem alguma capacitação pedagógica em MAP

$\begin{array}{lll}\text { Não } & 29 & 46,0 \\ \text { Sim } & 34 & 54,0\end{array}$

Capacitação pedagógica realizada em MAP foi****

Oferecida pela UFSC $19 \quad 57,6$

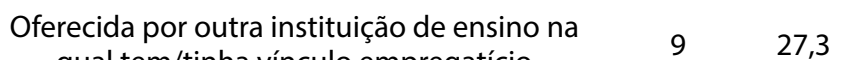
qual tem/tinha vínculo empregatício

$12 \quad 36,4$ área por conta própria

Outra

$6 \quad 17,5$

Continua... 
Tabela 2. (Continuação) Descrição da amostra segundo variáveis relacionadas às metodologias ativas de ensinoaprendizagem (MAP) na prática pedagógica dos docentes - Florianópolis e Araranguá, SC, 2021.

\begin{tabular}{ccc}
\hline Variável & N & $\%$ \\
\hline $\begin{array}{c}\text { Capacitação realizada foi suficiente para dar densidade teórica ao } \\
\text { processo de ensino-aprendizagem com MAP** }\end{array}$ & 23 & 67,7 \\
Não & 11 & 32,3 \\
Sim & 22 & 34,9 \\
Avaliação da própria capacitação pedagógica em MAP \\
Ótima/boa \\
Regular \\
Ruim/péssima & 28 & 44,4 \\
1 ou 2 & 13 & 20,6 \\
3 ou mais & 18 & 31,6 \\
\end{tabular}

*Mais de uma opção poderia ser assinalada por cada participante, e o percentual foi calculado a partir dos que responderam "sim" à questão sobre realização de capacitação em MAP.

**Somente responderam aqueles que tinham como resposta "sim" à questão sobre realização de capacitação em MAP.

***Somente responderam aqueles que tinham como resposta "sim" à questão sobre uso de MAP na prática docente.

Fonte: Elaborada pelos autores. de simulação (31,7\%). Educação entre pares foi a MAP menos conhecida (33,3\%) e utilizada (7,9\%) pela amostra (Figura 2).

Foram testadas associações entre as características demográficas, de formação acadêmica e de atuação docente com as variáveis relacionadas à utilização e capacitação docente em MAP. Não houve diferença em relação a campus de atuação, anos de atuação como docente, curso de formação na graduação, titulação ou regime de trabalho com o uso ou não de MAP, dificuldade no seu uso ou realização de capacitação pedagógica no tema (dados não apresentados). A única associação encontrada foi em relação ao número de MAP utilizadas com apresentar dificuldades no seu uso na prática docente. Os docentes que referiram ter dificuldade com MAP acabam utilizando de uma a duas MAP $(55,2 \%)$, enquanto aqueles que não têm dificuldades relatam utilizar três ou mais diferentes MAP na sua prática docente $(92,9 \%)(p<0,001)$.

\section{DISCUSSÃO}

Os resultados deste estudo apontam o seguinte: 1. a maioria dos docentes refere que utiliza ou já utilizou MAP na sua atuação como docente, e metade deles refere ter alguma dificuldade no seu uso; 2 . pouco mais da metade dos docentes já realizou alguma capacitação pedagógica nesse tema, e, entre os que realizaram, a maioria considera que a capacitação não foi suficiente para dar densidade teórica ao processo de ensino-

Figura 2. Percentual de conhecimento e utilização de diferentes metodologias ativas de ensino-aprendizagem (MAP) dos docentes do curso de Medicina dos campi de Florianópolis e Araranguá da Universidade Federal de Santa Catarina Florianópolis e Araranguá, SC, 2021.

Conhece ou já ouviu falar sobre Utiliza e/ou já utilizou na prática docente

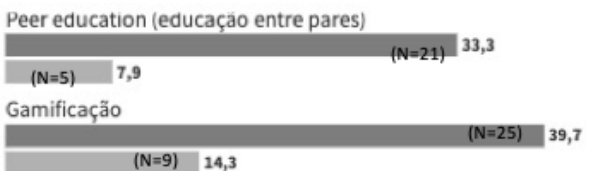

( $N=9$ ) 14,3

Aprendizagem baseada em projetos (Project-based learning - PjBL)

$(\mathrm{N}=8) \quad 12,7$

Aprendizagem baseada em equipes (Team-based learning - TBL)

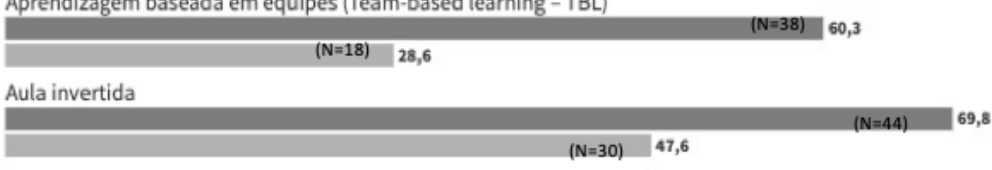

Laboratórios de simulação

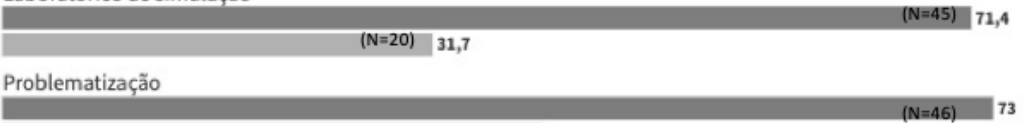

Problematização

(N=35) 39,7

Aprendizagem baseada em problemas (Problem-based learning - PBL)

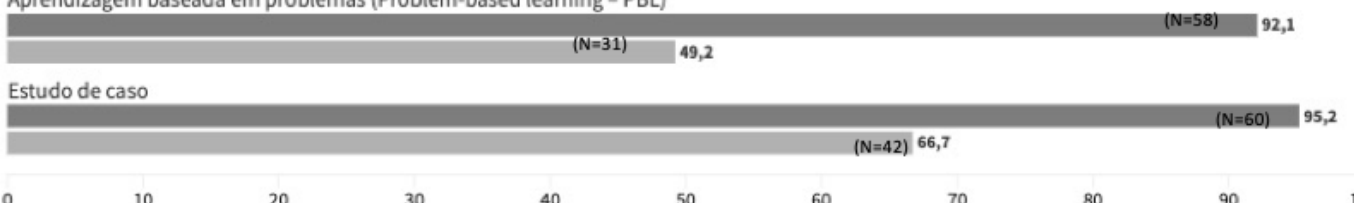

10

20

30

60

70

100

Fonte: Elaborada pelos autores. 
aprendizagem com MAP; 3. a maioria dos docentes utiliza/ utilizou três ou mais diferentes MAP, sendo estudo de caso e PBL as mais comuns; 4 . não foram encontradas diferenças entre os aspectos de MAP avaliados nos dois campi da universidade.

Primeiramente, a amostra do presente estudo conta com diferenças em relação a outras publicações que contêm dados do perfil docente em Medicina em universidades federais. Em relação ao regime de trabalho, a presente pesquisa contou com a maioria dos docentes em dedicação exclusiva, ao contrário do que foi apontado no estudo de Ferreira e Souza $(2016)^{13}$, em que havia mais docentes (80\%) com contrato parcial de 20 horas. Este estudo corrobora o relatório da Comissão Interinstitucional Nacional de Avaliação do Ensino Médico (Cinaem) ${ }^{14}$, segundo o qual a minoria dos docentes de Medicina se dedica exclusivamente à docência. Quanto ao sexo, a maior parte das publicações conta com proporção maior de homens ${ }^{13-15}$, ao contrário do presente estudo de cuja amostra participaram $57 \%$ de mulheres. No que se refere à formação acadêmica, foi apontado que quase nove entre dez docentes tinham doutorado, percentual maior que o encontrado em outras universidades ${ }^{15,16}$.

Quanto à utilização de MAP na atuação como docente, este estudo teve prevalência maior $(90,5 \%)$ do que a relatada pela literatura. Na Universidade do Estado do Rio Grande do Norte (UERN), por exemplo, os métodos de ensino tradicionais, como aula expositiva, foram os mais relatados como usados entre os docentes (76\%), e MAP foram utilizadas por $35 \%$ da amostra de docentes do curso de Medicina da instituição. Entre eles, um a cada três afirmou que gostaria de utilizar diferentes metodologias de ensino, citando a capacitação pedagógica como um dos principais fatores limitantes ${ }^{17}$. De acordo com uma revisão sobre aspectos da docência na área da saúde no Brasil, há a predominância do ensino tradicional e pouco preparo pedagógico dos docentes, que acabam ficando distantes das necessidades que o trabalho em saúde exige ${ }^{11}$.

A educação permanente dos docentes é uma demanda apontada dentro das instituições de ensino superior (IES), além da necessidade de uma cultura de valorização do ensino ${ }^{11}$. Dentro da UFSC, 54\% dos docentes referiram ter realizado alguma capacitação pedagógica em MAP. Leite et al. ${ }^{18}$, em pesquisa realizada com docentes da Medicina da Universidade Federal de São João del-Rei (UFSJ), apontam que $41 \%$ deles realizaram algum tipo de preparação didáticopedagógica, o que inclui capacitações de professores. Se considerarmos que nesse percentual entrariam cursos das mais diferentes áreas e temas, a capacitação em MAP, se existente, foi em percentual menor que na presente pesquisa. As universidades, ao contratarem os docentes, costumam considerar aspectos de ensino e não de aprendizagem, e, nos próprios processos seletivos, o professor está no centro das informações e é avaliado pela transmissão de conhecimentos por meio de aulas expositivas tradicionais. Além disso, programas de capacitação docente permanente não fazem parte da maioria das instituições ${ }^{19}$.

Entre os docentes da UFSC que realizaram capacitação pedagógica em MAP, a maioria o fez por capacitação oferecida pela própria universidade, e um a cada três se inscreveu em algum curso por conta própria, o que mostra parecer haver um interesse deles nessa área e/ou uma necessidade relacionada a ela. No entanto, o dado de que a capacitação realizada não foi suficiente para dar densidade teórica ao processo de ensinoaprendizagem com MAP para a maioria dos docentes reforça que ainda existe uma lacuna de capacitação nesse tema dentro da universidade. Outros estudos que analisam capacitação pedagógica no ensino médico relatam a necessidade de grande carga horária e aprofundamento no tema para que possa ser utilizado no dia a dia com mais confiança ${ }^{20,21}$.

Estudo de caso e PBL foram as MAP mais conhecidas e utilizadas pelos docentes dentro da UFSC. A PBL é a MAP mais utilizada dentro dos cursos de Medicina ${ }^{22}$, o que pode explicar o motivo de nove entre cada dez docentes citarem que conhecem a metodologia e/ou já ouviram falar sobre ela.

A PBL surge na década de 1960 como tentativa de responder à mudança necessária da formação médica, alterando o formato de aula expositiva tão utilizado até então, sendo adotada inicialmente no Brasil a partir da década de 199022,23. O estudo de caso também é um método centrado na solução de um problema, e sua diferença quanto à $\mathrm{PBL}$, de maneira simplista, é o momento em que o estudante é apresentado ao problema e tem acesso aos conceitos envolvidos em cada caso ${ }^{23}$.

A literatura aponta melhor desempenho acadêmico entre alunos que trabalham em pequenos grupos por meio de MAP em cursos de graduação na área da saúde ${ }^{24}$. No entanto, para que as MAP estejam em consonância com o modelo curricular inovador preconizado pelas DCN, o corpo docente deve se dedicar integralmente à IES e estar capacitado. Além disso, é imprescindível que haja estrutura física adequadada ${ }^{22}$. Quando não existe uma consonância entre todas as esferas envolvidas e necessárias, as aulas tendem ao formato tradicional ${ }^{16}$.

Quanto se analisou a associação entre as características individuais dos docentes e as variáveis relacionadas às MAP, não se observaram, dentro da UFSC, diferenças estatísticas. Entre docentes da UERN, foram encontradas diferenças no uso de MAP de acordo com variáveis individuais, em que maior experiência docente, graduação em outros cursos da área da saúde diferentes da Medicina, maior tempo desde a graduação e titulação em nível de pós-graduação stricto sensu favoreceram seu uso ${ }^{17}$. 
Além das características individuais, foi avaliado se existiam diferenças entre os campi da UFSC em relação à adoção de MAP e dificuldades na realização de capacitação no tema. O curso de Medicina dentro da UFSC, como já citado anteriormente, estava em andamento em dois campi com realidades diferentes: em Florianópolis, o curso é mais antigo e o currículo passou por mudanças ao longo do tempo, e, em Araranguá, o curso teve início no segundo semestre de 2018, sendo já planejado no formato preconizado pelas atuais DCN.

Biffi et al. ${ }^{16}$, assim como no presente estudo, utilizaram dados de docentes que atuam na Medicina em duas realidades referentes às MAP: uma IES mais antiga que readaptou o projeto político-pedagógico e as MAP foram acrescentadas como metodologias de ensino, e outra IES criada mais recentemente teve como base as DCN de 2014 e, desde o início de funcionamento, já adotava as MAP. $\mathrm{O}$ estudo foi qualitativo, mas apontou maior conhecimento sobre a utilização das MAP entre os docentes da IES mais antiga.

Dentro da UFSC, o resultado de que não foram encontradas quaisquer diferenças entre os docentes nos dois campi mostra que, mesmo com realidades diferentes, as MAP vêm sendo utilizado em ambos os campi, os docentes têm a mesma proporção de realização de capacitação no tema, e, nos dois locais, são relatadas dificuldades no seu uso. Ressalta-se que o questionário não abordou a frequência de uso das MAP, não sendo possível dizer se eram utilizadas pelos docentes todo o tempo ou em momentos específicos durante o semestre. Não se analisou ainda se as dificuldades e capacitações realizadas eram as mesmas ou não.

\section{CONSIDERAÇÕES FINAIS}

O uso das MAP na prática pedagógica dos docentes de Medicina da UFSC é alto, e a maioria dos professores utiliza diferentes metodologias na sua atuação como docentes. Quanto à capacitação em MAP, a maioria já fez, mas ainda assim considera que existem dificuldades no seu uso com os discentes, o que demonstra que mudanças são necessárias, seja na metodologia, na carga horária ou nos temas das capacitações que são oferecidas. Mesmo com realidades bem diferentes na implantação do curso nos dois campi analisados, não foram encontradas diferenças relacionadas ao uso, à dificuldade e à capacitação em MAP entre os docentes.

\section{CONTRIBUIÇÃO DOS AUTORES}

Katia Jakovljevic Pudla Wagner foi responsável pelo desenho da pesquisa, pela estruturação do manuscrito, pela análise e interpretação dos resultados, e pela redação do manuscrito. Lourival José Martins Filho foi responsável pela redação e revisão crítica do manuscrito

\section{CONFLITO DE INTERESSES}

Declaramos não haver conflito de interesses.

\section{FINANCIAMENTO}

Declaramos não haver financiamento.

\section{REFERÊNCIAS}

1. Brasil. Resolução CNE/CES no 4, de 7 de novembro de 2001. Institui Diretrizes Curriculares Nacionais do Curso de Graduação em Medicina. Diário Oficial da União; 9 nov 2001. Seção 1, p. 38.

2. Brasil. Resolução no 3, de 20 de junho de 2014. Institui Diretrizes Curriculares Nacionais do Curso de Graduação em Medicina e dá outras providências. Diário Oficial da União; 23 jun 2014. Seção 1, p. 8-11.

3. Nagai WA, Izeki CA. Relato de experiência com metodologia ativa de aprendizagem em uma disciplina de programação básica com ingressantes dos cursos de Engenharia da Computação, Engenharia de Controle e Automação e Engenharia Elétrica. Rev RETEC. 2013;4:1-10.

4. Hissaki T. Aprender e ensinar na escola vestida de branco. São Paulo: Phorte; 2010. 240 p.

5. Mitre SM, Siqueira-Batista R, Girardi-de-Mendonça JM, de MoraisPinto NM, Meirelles CAB, Pinto-Porto $C$, et al. Active teaching-learning methodologies in health education: current debates. Cien Saude Colet. 2008;13(Suppl 2):2133-44.

6. Wall ML, Prado ML, Carraro TE. A experiência de realizar um estágio docência aplicando metodologias ativas. Acta Paul Enferm. 2008;21(3):515-9.

7. Meireles MAC, Fernandes CCP, Silva LS. Novas Diretrizes Curriculares Nacionais e a formação médica: expectativas dos discentes do primeiro ano do curso de Medicina de uma instituição de ensino superior. Rev Bras Educ Med. 2019:43(2):67-78.

8. Custódio JB, Peixoto MGB, Arruda CAM, Vieira DVF, Sousa MSD, Ávila MMM. Desafios associados à formação do médico em saúde coletiva no curso de Medicina de uma universidade pública do Ceará. Rev Bras Educ Med. 2019;43(2):114-21.

9. Mesquita SKC, Meneses RMV, Ramos DKR. Metodologias ativas de ensino/ aprendizagem: dificuldades de docentes de um curso de enfermagem. Trab Educ Saúde. 2016;14(2):473-86.

10. Rêgo C, Batista SH. Desenvolvimento docente nos cursos de Medicina: um campo fecundo. Rev Bras Educ Med. 2012;36(3):317-24.

11. Silva VOD, Pinto ICM. Produção científica sobre docência em saúde no Brasil. Saúde Debate. 2019;43:134-47.

12. Universidade Federal de Santa Catarina. UFSC: estrutura [acesso em 31 ago 2021]. Disponível em: https://estrutura.ufsc.br/.

13. Ferreira CCS, Souza AML. Formação e prática do professor de Medicina: um estudo realizado na Universidade Federal de Rondônia. Rev Bras Educ Med. 2016;40(4):635-43.

14. Comissão Interinstitucional Nacional de Avaliação do Ensino Médico Avaliação das escolas médicas brasileiras - relatório geral. Rio de Janeiro: Cinaem; 1997.

15. Garcia MAA, Silva ALB. Um perfil do docente de medicina e sua participação na reestruturação curricular. Rev Bras Educ Med. 2011;35(1):58-68.

16. Biffi M, Diercks MS, Barreiros BC, Fajardo AP. Active learning methodologies: challenges for professors of two medical schools in Rio Grande do Sul, Brazil. Rev Bras Educ Med. 2020; 44(04), e145.

17. Cruz POD, Carvalho TB, Pinheiro LDP, Giovaninni PE, Nascimento EGC Fernandes TAAM. Percepção da efetividade dos métodos de ensino utilizados em um curso de Medicina do Nordeste do Brasil. Rev Bras Educ Med. 2019;43(2):40-7.

18. Leite VT, Vazzi PIFL, Moura MBR, Pereira LS, Caldas Neto TP, Lima EHM. Avaliação do perfil dos professores de Medicina de uma universidade do interior de Minas Gerais. Rev Bras Educ Med. 2020;44(3):e096. 
19. Machado JLM, Machado VMV, Joaquim E. Formação e seleção de docentes para currículos inovadores na graduação em saúde. Rev Bras Educ Med. 2011;(35)3:326-33.

20. Al Wardy N. Faculty Development Initiatives at the College of Medicine \& Health Sciences, Sultan Qaboos University, Muscat, Oman. Sultan Qaboos Univ Med J. 2020;20(3):e271-e8.

21. van Diggele $C$, Burgess $A$, Mellis $C$. Teacher training program for medical students: improvements needed. Adv Med Educ Pract. 2015;6:265-70.
22. Machado CDB, Wuo A, Heinzle M. Educação médica no Brasil: uma análise histórica sobre a formação acadêmica e pedagógica. Rev Bras Educ Med. 2018;42(4):66-73.

23. Albanese MA, Mitchell S. Problem-based learning: a review of literature on its outcomes and implementation issues. Acad Med. 1993;68(1):52-81.

24. Kalaian SA, Kasim RM. Effectiveness of various innovative learning methods in health science classrooms: a meta-analysis. Adv Health Sci Educ Theory Pract. 2017;22(5):1151-67. 